\title{
1 Habitat choice stabilizes metapopulation dynamics through increased ecological specialisation.
}

2 Frederik Mortier ${ }^{1}$, Staffan Jacob $^{2,3}$, Martijn L. Vandegehuchte ${ }^{1}$, Dries Bonte ${ }^{1}$

31 Terrestrial Ecology Unit, Department of Biology, Ghent University, Karel Lodewijk

$4 \quad$ Ledeganckstraat 35,9000 Ghent, Belgium

52 Station d'Ecologie Théorique et Expérimentale, CNRS UMR5321, 09200, Moulis, France

$6{ }^{3}$ Université Catholique de Louvain, Earth and Life Institute, Biodiversity Research Centre, Croix

7 du Sud 4, L7-07-04, 1348 Louvain-la-Neuve, Belgium

8 Corresponding author: Frederik Mortier, email: fremorti.mortier@UGent.be

Author contributions: FM developed the model, analysed and interpreted results, drafted the manuscript an was involved in discussions at all stages; DB supervised the development of the model, was involved in interpreting the results, provided critical revisions of the manuscript and was involved in discussions at all steps; SJ interpreted the results, provided critical revisions of the manuscript and was involved in discussions at all steps; MV interpreted the results, provided critical revisions of the manuscript and was involved in discussions from the analysis onward.

Acknowledgements: We would like to thank Pim Edelaar, François Massol and Estelle Laurent for engaging in insightful discussions. The project received financial support from the Research Foundation - Flanders (FWO) through research grant G.018017.N (FM, MVG \& DB) and the research network EVENET (DB, SJ).

\section{Open data:}

21 All code is available at https://github.com/fremorti/Habitat_choice_stabilizes_metapopulations

\section{Competing interests:}

23 All authors declare to have no competing interests. 


\section{Abstract}

26 Dispersal is a key trait responsible for the spread of individuals and genes among local populations, 27 thereby generating eco-evolutionary interactions. Especially in heterogeneous metapopulations, a tight

28 coupling between dispersal, population dynamics and the evolution of local adaptation is expected. In 29 this respect, current theory predicts dispersal to counteract ecological specialisation by redistributing 30 locally selected phenotypes (i.e. migration load). However, in nature we observe that some specialists 31 exhibit a strong dispersal capacity.

32 Habitat choice following informed dispersal decisions, provides a possible mechanism for individuals 33 to match the environment to their phenotype, thereby enabling the persistence of evolved ecological 34 specialisation. How such informed decisions affect the evolution of dispersal and ecological 35 specialisation and how these, in turn, influence metapopulation dynamics is yet to be determined.

36 By means of individual-based modelling, we show that informed decisions on both departure and settlement decouples the evolution of dispersal and generalism, favouring highly dispersive specialists.

38 Choice at settlement decouples dispersal from ecological specialisation most effectively. Additionally, 39 habitat choice stabilizes local and metapopulation demography because of the maintenance of 40 ecological specialisation at all levels of dispersal propensity.

41 We advocate considering habitat choice in spatially structured ecological models to improve demographic predictions in the face of environmental change.

43 Key-words: habitat choice, spatial ecology, dispersal, ecological specialisation, local adaptation, ecoevolutionary dynamics 
45

\section{Introduction}

Most populations are spatially structured and organized in metapopulations. Local populations are connected by dispersal, the movement of individuals or propagules that potentially generates gene flow across space [1]. Dispersal thus acts as a glue that links local gene pools, local population dynamics and metapopulation dynamics [2]. Dispersal is known to evolve in response to metapopulation structure as a bet-hedging strategy in spatio-temporally variable environments $[1,3-5]$. It is also known to evolve in response to local drivers, for instance to escape kin competition and inbreeding depression [1,3]. Dispersal is thus an essential attribute for fitness maximisation [6]. Ultimately, these benefits are balanced against dispersal costs to determine the optimal dispersal strategy -in terms of frequency and distance.

In metapopulations, habitat heterogeneity introduces additional costs to dispersal. In such a setting, divergent selective pressures among local habitats can result in local adaptation [7]. Often, local adaptation comes at the cost of lower performance in other environments, which can lead populations to adapt to a smaller subset of available habitats -i.e. ecological specialisation [8]. If specialists experience the landscape as highly heterogeneous when dispersing [9], they are more likely to end up in unsuitable habitat. Ecological specialisation is therefore predicted to select against dispersal $[10,11]$. Reciprocally, dispersal hinders local adaptation by mixing gene pools and thus opposes the evolution of specialisation [12-15]. This trade-off between dispersal and local adaptation is expected to maximize performance across an environmentally heterogeneous landscape, and thus homogenize fitness. High dispersal rates and ecological specialisation are, therefore, difficult to reconcile (theoretical evidence: [11,15-19], empirical evidence: [12,13,20]).

If dispersal involves habitat choice, dispersal and ecological specialisation may be reconcilable. From a pure movement perspective, optimal foraging theory inherently implements choice-based movement, with individuals weighing the cost of foraging time against the benefit of finding the optimal resource [21]. Such adaptive behaviour could also be relevant in the context of dispersal -when considering movements directly linked to reproduction. Rather than assuming that each member of a population disperses randomly with the same probability, informed dispersal implies a non-random subset of the 
local population dispersing and/or dispersers being redistributed in a non-random way across the landscape [22-25]. Individuals can indeed use information about their phenotype and environment to decide whether to disperse (departure decision), and where to go (settlement decision; [26]). Several organisms gather and use information during movement [27-29]. Intuitive in the light of evolution, habitat choice enables individuals to track the environment that best matches their phenotype while also reaping the benefits of dispersal [30,31]. Furthermore, theoretical and experimental studies show how habitat choice cascades into further life-history evolution, increasing local adaptation and ecological specialisation (Theory: [32-36]; empirical: [22,37]).

With habitat choice, individuals integrate information to preferentially disperse towards habitat that maximizes their fitness, which affects the evolution of dispersal and specialisation. Subsequently, rapid evolutionary changes are expected to affect ecological dynamics in the same time frame according to the eco-evolutionary framework [38]. Understanding changes in metapopulation dynamics should enable us to understand and predict metapopulation persistence in a spatially structured and heterogeneous context. Here, we develop an individual-based model to study the impact of habitat choice on the evolution of dispersal and ecological specialisation. We separately analyse 1) how dispersal and habitat choice affect the evolution of ecological specialisation, and 2) how ecological specialisation and habitat choice affect dispersal evolution. Furthermore, we quantified the consequences of these evolutionary processes for metapopulation dynamics. While random dispersal should lead to a trade-off between dispersal and ecological specialisation, we predict habitat choice to favour specialists, even with high levels of dispersal. Additionally, we predict that habitat choice favours high levels of dispersal, even in specialists. Moreover, dispersal can affect how local populations within a metapopulation vary over time, increasing or decreasing metapopulation stability and synchrony [39-41]. We predict that the individual fitness maximisation by habitat choice should likewise favour the metapopulation by resulting in an increased metapopulation size and stability. Finally, since the consequences of habitat choice for ecological and evolutionary dynamics have been predicted to differ depending on the timing of the informed decision [42], we modelled four dispersal 
bioRxiv preprint doi: https://doi.org/10.1101/267575; this version posted February 19, 2018. The copyright holder for this preprint (which was not certified by peer review) is the author/funder, who has granted bioRxiv a license to display the preprint in perpetuity. It is made available under aCC-BY-NC-ND 4.0 International license.

98 scenarios: random dispersal, habitat choice at departure, habitat choice at settlement, and habitat 99 choice at both departure and settlement. 


\section{Landscape}

102 We model a finite landscape: a toroidal lattice of $32 \times 32$ grid cells. Each patch (i.e. grid cell) has a 103 random environmental value $v_{x, y} \in[0,1]$ at coordinates $\mathrm{x}, \mathrm{y}$, without any spatial autocorrelation.

104 This environmental value is the local selective pressure. Its values are randomly distributed in space 105 and constant in time creating a heterogeneous environment. Additionally, each patch contains a certain 106 amount of resources $\left(G_{x, y}\right)$ that regulate local consumer population densities.

109 For simplicity, we model an asexually reproducing organism with discrete generations. The sequence of life-history events for each individual starts with dispersal and is succeeded by reproduction, then population regulation. This closely resembles soft selection in a semelparous species with a single dispersal phase [43]. These life-history events are explained below and assumed parameters are summarized in table 1.

116 We model three traits that can be allowed to evolve:

117 The optimal habitat trait $(\boldsymbol{m u T})$ determines the optimum of the environmental value $\left(v_{x, y}\right)$ for an

118 individual to have its highest possible fitness $(m u T \in[0,1])$.

119 The niche width (varT) determines the extent of ecological specialisation, by determining an individual's fitness for values of the environment $\left(v_{x, y}\right)$ a certain distance away from the individual's optimal habitat $(m u T)$. A wide niche results in higher fitness far away from the optimal habitat (eq. 2;

122 derived from Chaianunporn \& Hoverstad [19], but decreases fitness in the optimal habitat (eq. 3, fig.

123 1). The match of an individual's optimal habitat $(m u T)$ with the local environmental value $\left(v_{x, y}\right)$ combined with its varT determines the individual's efficiency in this particular habitat $\left(\alpha_{i}\right.$, eq. 2$)$. The 
125 amount of gathered resources $\left(F_{i}\right)$ combines the individual's efficiency $\left(\alpha_{i}\right)$ with the local resource

126 density $\left(R_{x, y}\right)$ and is proportional to the individual's expected reproductive success (eq. 1).

127

138

1) $F_{i}=\frac{\alpha_{i} * R_{x, y}}{1+h\left(1+\alpha_{i} * R_{x, y}\right)}$

2) $\alpha_{i}=a_{\max } * \gamma_{i} * e^{-\frac{\left(m u T_{i}-v_{x, y}\right)^{2}}{\operatorname{varT}_{i}^{2}}}$

3) $\gamma_{i}=e^{-c t * \operatorname{var} T_{i}}$

$F_{i}$ represents the gathered resources by individual $\mathrm{i}$, determined by a resource-consumer model with $\mathrm{h}$ being handling time, $R_{x, y}$ the amount of resources present locally, $a_{\max }$ the maximum encounter rate, $m u T_{i}$ and $\operatorname{var} T_{i}$ being the optimal habitat trait and niche width respectively for that individual and $v_{x, y}$ the local environmental value. $\gamma_{i}$ implements the niche width-performance trade-off with $c t$ indicating the strength of the trade-off.

The dispersal trait (d) represents the individual's inclination to disperse. The role of this trait is explained in more detail in the next section.

\section{Dispersal}

Individuals disperse before selection occurs. We model two decision points in a dispersal event:

First, at departure, an individual disperses with a probability equal to its dispersal trait (d) if departure is random, meaning that a higher dispersal trait implies a higher tendency to disperse. With departure choice, the dispersal trait (d) represents the minimal acceptable local expected reproductive output at which an individual chooses not to disperse. Below this threshold, the local conditions are considered too bad and the individual leaves. In parallel with random departure, a higher threshold implies a higher tendency to disperse.

At settlement, an individual settles in a random patch within its dispersal range determined by a maximum dispersal distance $(d r)$ if settlement is random (all patches $x_{i}^{\prime}, y_{i}^{\prime} \mid x_{i}^{\prime} \in\left[x_{i}-d r, x_{i}+\right.$ $\left.d r] ; y_{i}^{\prime} \in\left[y_{i}-d r, y_{i}+d r\right]\right)$. Its current location is excluded from this range to force dispersing individuals to change location. With habitat choice at settlement, the dispersing individual settles in 
151 the location where the local environmental value $\left(v_{x, y}\right)$ best matches its own optimal habitat trait

$152(m u T)$ within its dispersal range.

153 Note that habitat choice at both decision points involves evaluating how well the individual's optimal

154 habitat $(m u T)$ trait matches the environmental value of a patch $\left(v_{x, y}\right)$ [24].

157 Reproducing individuals have an expected number of offspring $\left(\lambda_{i}\right)$ proportional to their gathered 158 resources $\left(F_{i}\right)$ in the patch after the dispersal phase.

$\sigma$ Indicates how many offspring each unit of resources results in. The actual reproductive output of an individual is sampled from a Poisson distribution with mean $\lambda_{i}$.

\section{Local population regulation}

163 Local consumer populations are regulated through local resource availability $\left(G_{x, y}\right)$. These resources

164 are restocked each generation according to a logistic growth function,

$$
\delta G_{x, y}=R_{G} *\left(1-\frac{G_{x, y}}{K_{G}}\right)
$$

where the local resource increase $\delta G_{x, y}$ depends on $G_{x, y}$, the amount of resources already present

166 locally. Furthermore, $R_{G}$ and $K_{G}$ represent the optimal growth rate and carrying capacity of the

167 resources respectively. Resources are consumed proportionally to the number of offspring. A

168 consumer's offspring without the required amount of resources in their local patch will die after

169 depleting the leftover resources should there be any left. Population regulation depends on resource 170 availability and, consequently, is density dependent, while habitat choice is decoupled from local

171 population densities. Hereby, we avoid simulating density-dependent habitat choice (see [31] for a 172 comparison of different modes of habitat choice). 
175 Non-fixed traits mutate at a rate of 0.01 generation $^{-1}$. The optimal habitat trait $(m u T)$, dispersal trait $(d)$

176 and niche width (varT) mutate by randomly sampling a new trait value from a normal distribution with

177 the initial trait value as mean and standard deviation 0.1 . New values of $m u T$ are limited to the range

178 of possible environmental values $[0,1]$.

\section{Simulations}

180 We analyse four models that represent all combinations of either random dispersal or habitat choice at 181 departure and at settlement. First, we analyse how niche width (varT) evolves for different fixed 182 values of the dispersal trait (d) and the different habitat choice scenarios. Second, we analyse how 183 dispersal evolves for different fixed values of niche width with the different combinations of habitat 184 choice. Fixed traits are varied over 20 values with equal increments within a range (i.e. random 185 departure $\mathrm{d}:[0,1]$, informed departure $\mathrm{d}:[0,5], \operatorname{var} T:[0,0.5])$. The range of the dispersal trait (d) in 186 informed departure scenarios differs from the random scenarios since it represents the minimal 187 acceptable reproductive output of an individual instead of its dispersal propensity. This range of $\mathrm{d}$ in 188 the informed departure scenarios, however, results in actual dispersal propensities that cover the range $189[0,1]$. Each scenario is replicated 10 times with each replicate simulated for 500 generations. We 190 analyse average niche width of the last generation in scenarios with evolving niche width, and the 191 proportion of individuals that dispersed in the last generation in scenarios with the dispersal trait 192 evolving. Additionally, we analyse the metapopulation size (i.e. total number of individuals) in the last 193 generation and temporal variability in local population sizes, temporal variability in metapopulation 194 size and asynchrony ( $\alpha-, \gamma$ - and $\beta$-variability respectively [44], in the last five generations for each 195 replicate.

\section{Initialisation}

198 We initialize each replicate by allocating 70000 individuals randomly across the $32 \times 32$-landscape grid. This initial metapopulation size close to the consumers' carrying capacity avoids drift effects. Each individual's optimal habitat trait value is sampled from a uniform distribution between 0 and 1 . 
201 Unless it was fixed for that scenario, niche width and the dispersal trait are randomly sampled from a

202 uniform distribution (with the same range as their fixed values for the fixed scenarios).

203 Imperfect habitat choice

204 In addition to the analyses presented in this manuscript, we tested whether imperfect rather than

205 perfect choice (at departure and settlement) leads to different eco-evolutionary dynamics (see

206 supplementary material 2). We modelled imperfect choice as a probability, at each decision point, that

207 the individual chooses randomly instead of in an informed way.

Table 1: Assumed parameters

\begin{tabular}{|c|c|c|}
\hline $\boldsymbol{R}_{G}$ & Optimal resource growth rate & 0.25 \\
\hline$K_{G}$ & Resource carrying capacity & 1 \\
\hline $\boldsymbol{h}$ & Handling time & 0.2 \\
\hline$a_{\max }$ & Maximum encounter rate & 0.05 \\
\hline \multirow[t]{2}{*}{$c t$} & Cost of generalism & 1 \\
\hline & mutation rate & 0.01 generation $^{-1}$ \\
\hline$d r$ & Dispersal range & 2 \\
\hline$\sigma$ & Resource conversion factor & 300 \\
\hline
\end{tabular}




\section{Results}

\section{Niche width evolution}

210 With random dispersal (fig. 2: orange crosses), we find a gradual increase of niche width (i.e.

211 ecological generalism) when increasing dispersal propensity. This means that, in accordance with

212 classical predictions [11,15], a low dispersal propensity favours specialism while a high dispersal

213 propensity leads to the evolution of generalists. Habitat choice at departure results in a pattern

214 comparable to that of the uninformed model, with a low level of dispersal in specialists or a high level

215 of dispersal in generalists (fig. 2: orange circles). However, habitat choice at departure enables

216 specialism to still evolve at a higher dispersal propensity than in scenarios of random dispersal. The

217 inflexion point shifts from a dispersal propensity of around 0.35 to one of around 0.65 . In contrast,

218 habitat choice at settlement favours specialism in all scenarios (fig. 2: green crosses), indicating that

219 specialism evolves regardless of dispersal propensity. Additionally, a departure decision results in a

220 trend of even stronger specialism than if specialism evolves when a settlement decision is made. A

221 combination of departure and settlement decision shows specialism evolving in all scenarios but more

222 strongly so at lower dispersal propensities (fig. 2: green circles).

223 Regarding ecological dynamics, we find that increasing dispersal propensity results in a decreased

224 metapopulation size when dispersal is random or only informed at departure (fig. 3). Metapopulations

225 exhibiting a low level of dispersal as well as those in which individuals have informed settlement

226 achieve larger metapopulations (fig. 3) with more stable local populations ( $\alpha$, fig. 4). Note that those

227 scenarios with lower metapopulation sizes correspond with scenarios in which generalism evolved. If

228 dispersers only choose at departure, metapopulation sizes decrease to a minimum around the switch

229 from specialism to generalism (fig. 3: orange circles). Also, the very dispersive scenarios with

230 informed settlement result in local population variability being equal to or higher than without

231 settlement decision, while metapopulation sizes are still markedly higher (fig. 3-4). Metapopulation

232 variability $(\gamma)$ and asynchrony $(\beta)$ did not show clear trends (see supplementary material 1 ). 


\section{Dispersal evolution}

234 With random dispersal, dispersal propensity increases with niche width as predicted, but overall levels

235 of dispersal propensity are low (fig. 5, left panel, orange crosses). With a departure decision, dispersal

236 is also relatively infrequent (fig. 5, left panel, orange circles). In this case, however, dispersal

237 propensity decreases with niche width, resulting in the most specialized individuals being the most

238 dispersive (fig. 5, right panel, orange circles). With a departure choice, only mismatched individuals

239 disperse (i.e. individuals whose optimal habitat trait differs significantly from the habitat value of their

240 location). Since specialists only have a limited range of suitable habitats, they are less likely than

241 generalists to find themselves in sufficiently suitable habitat. Therefore, specialists benefit more from

242 dispersal with a departure decision. With a settlement decision, dispersal propensity is markedly

243 higher (fig. 5, green crosses). Here, dispersal propensity drops slightly in metapopulations of the most

244 specialized individuals (i.e. lowest niche width). With a decision both at departure and settlement,

245 dispersal propensity is relatively high (fig. 5, green circles). It is slightly lower than in scenarios with

246 only| a settlement decision because the additional departure decision prevents a proportion of the

247 metapopulation from dispersing. In this scenario, only the very specialized individuals exhibit a

248 significant decrease in tendency to disperse.

249 With increasing niche width, metapopulation size decreases (fig. 6) and local population variability

250 increases ( $\alpha$, fig. 7). We find no noteworthy effect of the departure decision nor the settlement decision

251 on metapopulation sizes. Local population variability does not show a strong effect of any of the

252 habitat choice scenarios either, except for the slightly more variable local populations with a

253 settlement choice. Metapopulation variability $(\gamma)$ and asynchrony $(\beta)$ did not show clear trends (see

254 supplementary material 1).

\section{Imperfect habitat choice}

256 For imperfect habitat choice, we found patterns intermediate to those of random and perfectly

257 informed scenarios (see supplementary material 2), except in metapopulations of generalists that 258 disperse most when having imperfect information. 
259

260

261

262

263

264

265

266

267

268

269

270

271

272

273

274

275

276

277

278

279

280

281

282

283

284

285

\section{Discussion}

Altering dispersal from a random process to one with habitat choice enables a reconciliation between ecological specialisation and dispersal evolution, as predicted. By allowing the evolution of ecological specialisation, habitat choice furthermore causes local populations to fluctuate less and metapopulation to reach higher sizes. Metapopulation-level performance is thus enhanced by habitat choice.

In accordance with previous studies, random dispersal leads to a trade-off between dispersal and ecological specialisation in the absence of habitat choice. Habitat choice changes this relationship to favour specialists even with high levels of dispersal and favour dispersal even in specialists. Our result confirms predictions from early theory $[25,45]$, as well as conclusions from more dedicated models with habitat choice either at departure [35,36] or at settlement [32-34]. We take this one step further by contrasting departure and settlement decisions and demonstrating that choice mechanisms at settlement favour specialized strategies for even higher levels of dispersal than choice mechanisms at departure. A probable explanation for this stronger effect of settlement decision is that habitat choice at settlement integrates information from all potential settlement locations, rather than only from the natal location with informed departure. We show that a departure choice enables the evolution of even more specialized strategies when specialists are favoured. Dispersal from an already suitable habitat is costlier in more specialized strategies. Informed departure -i.e. informed philopatry- benefits specialists most by retaining individuals in their suitable habitat and enabling individuals in unsuitable habitat to disperse. Similar patterns were found in studies on habitat autocorrelation (discussed in [9]). When heterogeneity in habitat is experienced as coarse-grained relative to the scale of dispersal, the imposed landscape structure is more likely to match individuals to a suitable habitat, but when it is fine-grained, mismatches tend to prevail. Habitat matching by the spatial structure, instead of habitat choice behaviour, can limit maladaptive gene flow and select for increased specialism [9]. No matter how habitat matching is attained, we suspect that restricting movement of phenotypes to similar types of habitat always has the potential to favour specialism.

Habitat choice enables the evolution of dispersive specialists and altered metapopulation dynamics. Our model shows that metapopulation size is directly determined by the level of specialisation and 
only indirectly by information use during departure or settlement. This explains why the relation

287 between metapopulation sizes and dispersal is inverse to that between evolved niche width and

288 dispersal in scenarios of fixed dispersal. When generalism is favoured -i.e. under high levels of random settlement- the optimal strategy results in smaller metapopulation sizes despite the unaltered

290 resource availability in our model. Individual interests do not necessarily line up with those of the 291 metapopulation [46]. In addition, we show that local population sizes fluctuate more in 292 metapopulations with more generalists, which also have smaller metapopulation sizes. Small 293 metapopulations and high local population variability $(\alpha)$, which lead to more frequent occurrences of small local populations, increase the risk of extinction via demographic and genetic stochasticity

295 [47,48]. In favouring specialisation, habitat choice indirectly affects ecological dynamics, alters ecoevolutionary feedbacks and, as a consequence, can affect metapopulation persistence.

297 By demonstrating the impact of habitat choice on eco-evolutionary dynamics, our results question the 298 validity of the numerous models assuming random dispersal [34,49]. Dispersal without control during any phase likely applies to very few real systems such as bacteria and wind-dispersed plants. Many organisms are capable of more than just non-random dispersal, and move in a selective way, illustrated by obvious examples of habitat choice in grasshoppers [27,28], cue-based preferences during movement [50] and habitat choice [24,51,52]. Selective movement is even found in organisms for which it seems less obvious, such as zoochorous plants that disperse their seeds to suitable habitat via animals [53] or plankton that drift on currents but are able to select where to settle [54,55]. With the effect of habitat choice on ecological dynamics in mind, we recommend considering habitat choice when predicting the dynamics of spatially structured populations [23,34,42,49] -e.g. species distribution models, meta-community models, viability models. Our results show that in systems with habitat choice, assuming random dispersal may overlook habitat-choice effects on ecological specialisation, metapopulation size and stability. 
313 information can itself be a subject of selection. Organisms should rarely be able to acquire high-

314 precision information because of its high cost while a minimum of information is expected to be

315 adaptive [57]. We present results for a scenario of perfect information use, but even under imperfect

316 habitat choice our general evolutionary and ecological results stand (see supplementary material 2).

317 Different habitat-based choice behaviours that result in non-random dispersal may or may not differ in

318 their eco-evolutionary consequences [31,42]. Here, we show how the consequences of habitat choice

319 at departure differ from those of habitat choice at settlement. Habitat choice may also vary in several

320 other aspects, such as choice mechanisms and the reliability of information used [42,58]. We expect

321 such different variations on habitat choice to have additional influences on evolutionary and ecological

322 processes of varying magnitude. Hence, disregarding these nuances might conceal some crucial

323 insights or restrict us from generating detailed predictions. Unfortunately, we often lack information

324 on the specifics of habitat choice in real-life populations. Our focus going forward should be on

325 revealing the extent to which habitat choice varies along all these axes in nature, but also to evaluate

326 their relative importance.

327 In summary, we demonstrate the profound effect of habitat choice on eco-evolutionary dynamics of 328 metapopulations, including the dynamics of dispersal and ecological specialisation. Moreover, habitat 329 choice at settlement impacts the model's outcome more than choice at departure. Based on the 330 difference between random dispersal and habitat choice, we encourage studies of real-world 331 metapopulations to carefully consider habitat choice during the different phases of dispersal. Our 332 results elucidate the impact of the often erroneous assumption of random dispersal and may improve 333 the accuracy of future predictions of spatially structured populations.

336 1. Ronce, O. 2007 How Does It Feel to Be Like a Rolling Stone? Ten Questions About Dispersal 337 Evolution. Annu. Rev. Ecol. Evol. Syst. 38, 231-253. 
17. (doi:10.1111/j.1749-6632.2011.06419.x)

341 3. Bowler, D. E. \& Benton, T. G. 2005 Causes and consequences of animal dispersal strategies:

342 Relating individual behaviour to spatial dynamics. Biol. Rev. Camb. Philos. Soc. 80, 205-225.

$343 \quad$ (doi:10.1017/S1464793104006645)

344 4. McPeek, M. A. \& Holt, R. D. 1992 The Evolution of Dispersal in Spatially and Temporally

345 Varying Environments. Am. Nat. 140, 1010-1027. (doi:10.1086/285453)

346 5. Olivieri, I., Couvet, D. \& Gouyon, P. H. 1990 The genetics of transient populations: Research

347 at the metapopulation level. Trends Ecol. Evol. 5, 207-210. (doi:10.1016/0169-5347(90)90132-

$348 \quad$ W)

349 6. Bonte, D. \& Dahirel, M. 2017 Dispersal: a central and independent trait in life history. Oikos

$350 \quad$ 126, 472-479. (doi:10.1111/oik.03801)

351 7. Kawecki, T. J. \& Ebert, D. 2004 Conceptual issues in local adaptation. Ecol. Lett. 7, 1225-

352 1241. (doi:10.1111/j.1461-0248.2004.00684.x)

353 8. Poisot, T., Bever, J. D., Nemri, A., Thrall, P. H. \& Hochberg, M. E. 2011 A conceptual

354 framework for the evolution of ecological specialisation. Ecol. Lett. 14, 841-851.

$355 \quad$ (doi:10.1111/j.1461-0248.2011.01645.x)

$3569 . \quad$ Richardson, J. L., Urban, M. C., Bolnick, D. I. \& Skelly, D. K. 2014 Microgeographic

357 adaptation and the spatial scale of evolution. Trends Ecol. Evol. 29, 165-176.

$358 \quad$ (doi:10.1016/j.tree.2014.01.002)

359 10. Stevens, V. M. et al. 2014 A comparative analysis of dispersal syndromes in terrestrial and

360 semi-terrestrial animals. Ecol. Lett. 17, 1039-1052. (doi:10.1111/ele.12303)

361 11. Kisdi, E. 2002 Dispersal: Risk spreading versus local adaptation. Am. Nat. 159, 579-596.

$362 \quad($ doi:10.1086/339989)

363 12. Bolnick, D. I. \& Nosil, P. 2007 Natural selection in populations subject to a migration load.

364 Evolution (N. Y). 61, 2229-2243. (doi:10.1111/j.1558-5646.2007.00179.x)

365 13. Bolnick, D. I., Caldera, E. J. \& Matthews, B. 2008 Evidence for asymmetric migration load in 366 a pair of ecologically divergent stickleback populations. Biol. J. Linn. Soc. 94, 273-287.

$367 \quad($ doi:10.1111/j.1095-8312.2008.00978.x) 
368 14. Lenormand, T. 2002 Gene flow and the limits to natural selection. Trends Ecol. Evol. 17, 183-

369 189. (doi:10.1016/S0169-5347(02)02497-7)

370 15. Nurmi, T. \& Parvinen, K. 2011 Joint evolution of specialization and dispersal in structured

371 metapopulations. J. Theor. Biol. 275, 78-92. (doi:10.1016/j.jtbi.2011.01.023)

372 16. Nagelkerke, C. J. \& Menken, S. B. J. 2013 Coexistence of Habitat Specialists and Generalists 373 in Metapopulation Models of Multiple-Habitat Landscapes. Acta Biotheor. 61, 467-480.

$374 \quad$ (doi:10.1007/s10441-013-9186-4)

375 17. Billiard, S. \& Lenormand, T. 2005 Evolution of migration under kin selection and local

376 adaptation. Evolution (N. Y). 59, 13-23. (doi:10.1111/j.0014-3820.2005.tb00890.x)

377 18. Berdahl, A., Torney, C. J., Schertzer, E. \& Levin, S. A. 2015 On the evolutionary interplay

378 between dispersal and local adaptation in heterogeneous environments. Evolution (N. Y). 69,

379 1390-1405. (doi:10.1111/evo.12664)

380 19. Chaianunporn, T. \& Hovestadt, T. 2012 Concurrent evolution of random dispersal and habitat

381 niche width in host-parasitoid systems. Ecol. Modell. 247, 241-250.

$382 \quad$ (doi:10.1016/j.ecolmodel.2012.09.005)

383 20. Dahirel, M., Olivier, E., Guiller, A., Martin, M. C., Madec, L. \& Ansart, A. 2015 Movement

384 propensity and ability correlate with ecological specialization in European land snails:

385 Comparative analysis of a dispersal syndrome. J. Anim. Ecol. 84, 228-238. (doi:10.1111/1365-

$386 \quad 2656.12276)$

387 21. Charnov, E. L. 1976 Optimal foraging, the marginal value theorem. Theor. Popul. Biol. 9, 129-

388 136. (doi:10.1016/0040-5809(76)90040-X)

389 22. Rice, W. R. \& Salt, G. 1990 The Evolution of Reproductive Isolation as a Correlated Character

$390 \quad$ Under Sympatric Conditions: Experimental Evidence. Evolution (N. Y). 44, 1140-1152.

$391 \quad($ doi:10.2307/2409278)

392 23. Edelaar, P. \& Bolnick, D. I. 2012 Non-random gene flow: An underappreciated force in

393 evolution and ecology. Trends Ecol. Evol. 27, 659-665. (doi:10.1016/j.tree.2012.07.009)

394 24. Edelaar, P., Siepielski, A. M. \& Clobert, J. 2008 Matching habitat choice causes directed gene

395 flow: A neglected dimension in evolution and ecology. Evolution (N. Y). 62, 2462-2472. 
(doi:10.1111/j.1558-5646.2008.00459.x)

397

398

399

400

401

402

403

404

405

406

407

408

409

410

411

412

413

414

415

416

417

418

419

420

421

422

423

25. Holt, R. D. 1987 Population dynamics and evolutionary processes: the manifold roles of habitat selection. Evol. Ecol. 1, 331-347. (doi:10.1007/BF02071557)

26. Clobert, J., Le Galliard, J. F., Cote, J., Meylan, S. \& Massot, M. 2009 Informed dispersal, heterogeneity in animal dispersal syndromes and the dynamics of spatially structured populations. Ecol. Lett. 12, 197-209. (doi:10.1111/j.1461-0248.2008.01267.x)

27. Gillis, J. E. 1982 Substrate colour-matching cues in the cryptic grasshopper Circotettix rabula rabula (Rehn \&amp; Hebard). Anim. Behav. 30, 113-116. (doi:10.1016/S0003-3472(82)802443)

28. Ahnesjö, J. \& Forsman, A. 2006 Differential habitat selection by pygmy grasshopper color morphs; interactive effects of temperature and predator avoidance. Evol. Ecol. 20, 235-257. (doi:10.1007/s10682-006-6178-8)

29. Jacob, S., Chaine, A. S., Schtickzelle, N., Huet, M. \& Clobert, J. 2015 Social information from immigrants: Multiple immigrant-based sources of information for dispersal decisions in a ciliate. J. Anim. Ecol. 84, 1373-1383. (doi:10.1111/1365-2656.12380)

30. Cantrell, R. S., Cosner, C. \& Lou, Y. 2006 Movement toward better environments and the evolution of rapid diffusion. Math. Biosci. 204, 199-214. (doi:10.1016/j.mbs.2006.09.003)

31. Berner, D. \& Thibert-Plante, X. 2015 How mechanisms of habitat preference evolve and promote divergence with gene flow. J. Evol. Biol. 28, 1641-1655. (doi:10.1111/jeb.12683)

32. Bolnick, D. I. \& Otto, S. P. 2013 The magnitude of local adaptation under genotype-dependent dispersal. Ecol. Evol. 3, 4722-4735. (doi:10.1002/ece3.850)

33. Holt, R. D. \& Barfield, M. 2008 Habitat Selection and Niche Conservatism. Isr. J. Ecol. Evol. 54, 295-309. (doi:10.1560/IJEE.54.3-4.295)

34. Ravigné, V., Dieckmann, U. \& Olivieri, I. 2009 Live Where You Thrive: Joint Evolution of Habitat Choice and Local Adaptation Facilitates Specialization and Promotes Diversity. Am. Nat. 174, E141-E169. (doi:10.1086/605369)

35. Scheiner, S. M. 2016 Habitat Choice and Temporal Variation Alter the Balance between Adaptation by Genetic Differentiation, a Jack-of-All-Trades Strategy, and Phenotypic 
Plasticity. Am. Nat. 187, 000-000. (doi:10.1086/685812)

36. Armsworth, P. R. 2009 Conditional dispersal, clines, and the evolution of dispersiveness.

Theor. Ecol. 2, 105-117. (doi:10.1007/s12080-008-0032-2)

427

428

429

430

431

432

433

434

435

436

437

438

439

440

441

442

443

444

445

446

447

448

449

450

451

37. Jacob, S., Legrand, D., Chaine, A. S., Bonte, D., Schtickzelle, N., Huet, M. \& Clobert, J. 2017

Gene flow favours local adaptation under habitat choice in ciliate microcosms. Nat. Ecol. Evol. (doi:10.1038/s41559-017-0269-5)

38. Hendry, A. P. 2016 Eco-evolutionary dynamics. Princeton university Press.

39. Abbott, K. C. 2011 A dispersal-induced paradox: Synchrony and stability in stochastic metapopulations. Ecol. Lett. 14, 1158-1169. (doi:10.1111/j.1461-0248.2011.01670.x)

40. Taylor, A. D. 1990 Metapopulations, Dispersal, and Predator-Prey Dynamics: An Overview. Ecology 71, 429-433. (doi:10.2307/1940297)

41. Wang, S., Haegeman, B. \& Loreau, M. 2015 Dispersal and metapopulation stability. PeerJ 3, e1295. (doi:10.7717/peerj.1295)

42. Jacob, S., Bestion, E., Legrand, D., Clobert, J. \& Cote, J. 2015 Habitat matching and spatial heterogeneity of phenotypes: implications for metapopulation and metacommunity functioning. Evol. Ecol. 29, 851-871. (doi:10.1007/s10682-015-9776-5)

43. Massol, F. \& Débarre, F. 2015 Evolution of dispersal in spatially and temporally variable environments: The importance of life cycles. Evolution (N. Y). 69, 1925-1937. (doi:10.1111/evo.12699)

44. Wang, S. \& Loreau, M. 2014 Ecosystem stability in space: $\alpha, \beta$ and $\gamma$ variability. Ecol. Lett. 17, 891-901. (doi:10.1111/ele.12292)

45. Futuyma, D. J. \& Moreno, G. 1988 The Evolution of Ecological Specialization. Annu. Rev. Ecol. Syst. 19, 207-233. (doi:10.1146/annurev.es.19.110188.001231)

46. Del Mar Delgado, M., Ratikainen, I. I. \& Kokko, H. 2011 Inertia: The discrepancy between individual and common good in dispersal and prospecting behaviour. Biol. Rev. 86, 717-732. (doi:10.1111/j.1469-185X.2010.00167.x)

47. Lande, R. 1993 Risks of Population Extinction from Demographic and Environmental Stochasticity and Random Catastrophes. Am. Nat. 142, 911-927. (doi:10.1086/285580) 
452 48. Lande, R. 1994 Risk of Population Extinction from Fixation of New Deleterious Mutations.

453

454

455

456

457

458

459

460

461

462

463

464

465

466

467

468

469

470

471

472

473

474

475

476

477

478

479
Evolution (N. Y). 48, 1460. (doi:10.2307/2410240)

49. Travis, J. M. J. et al. 2012 Modelling dispersal: An eco-evolutionary framework incorporating emigration, movement, settlement behaviour and the multiple costs involved. Methods Ecol. Evol. 3, 628-641. (doi:10.1111/j.2041-210X.2012.00193.x)

50. Prokopy, R. J. 1968 Visual responses of apple maggot flies, rhagoletis pomonella (diptera: tephritidae): orchard studies. Entomol. Exp. Appl. 11, 403-422. (doi:10.1111/j.15707458.1968.tb02070.x)

51. Jaenike, J. \& Holt, R. D. 1991 Genetic Variation for Habitat Preference $\square$ : Evidence and Explanations. Am. Nat. 137, s67-s90.

52. Jacob, S., Legrand, D., Chaine, A. S., Bonte, D., Schtickzelle, N., Huet, M. \& Clobert, J. 2017 Gene flow favours local adaptation under habitat choice in ciliate microcosms. Nat. Ecol. Evol. 1, 1407-1409. (doi:10.1038/s41559-017-0269-5)

53. Spiegel, O. \& Nathan, R. 2010 Incorporating density dependence into the directed-dispersal hypothesis. Ecology 91, 1538-1548. (doi:10.1890/09-1166.1)

54. Burgess, S. C., Baskett, M. L., Grosberg, R. K., Morgan, S. G. \& Strathmann, R. R. 2016 When is dispersal for dispersal? Unifying marine and terrestrial perspectives. Biol. Rev. 91, 867-882. (doi:10.1111/brv.12198)

55. Bonte, D. et al. 2012 Costs of dispersal. Biol. Rev. 87, 290-312. (doi:10.1111/j.1469185X.2011.00201.X)

56. Cote, J., Bestion, E., Jacob, S., Travis, J., Legrand, D. \& Baguette, M. 2017 Evolution of dispersal strategies and dispersal syndromes in fragmented landscapes. Ecography (Cop.). 40, 56-73. (doi:10.1111/ecog.02538)

57. Bocedi, G., Heinonen, J. \& Travis, J. M. J. 2012 Uncertainty and the Role of Information Acquisition in the Evolution of Context-Dependent Emigration. Am. Nat. 179, 606-620. (doi:10.1086/665004)

58. Akcali, C. K. \& Porter, C. K. 2017 Comment on Van Belleghem et al. 2016: Habitat choice mechanisms in speciation and other forms of diversification. Evolution (N. Y). 71, 2754-2761. 


\section{Figures and tables:}

483 Figure 1: relation of local environmental value to individual efficiency in that location for a specialist (orange) and a generalist (blue) with optimal environment $(\mathrm{muT})=0.5$.

Figure 2: Niche width evolution in relation to effective dispersal propensity. Scenarios of random $(x)$

Figure 3: Metapopulation size for scenarios with fixed dispersal trait. Scenarios of random $(x)$ and informed ( $\odot$ ) departure combined with random (orange) or informed (green) settlement.

Figure 4: Local population variability for scenarios with fixed dispersal trait. Scenarios of random $(x)$ and informed (०) departure combined with random (orange) or informed (green) settlement.

Figure 5: Effective dispersal propensity resulting from an evolving dispersal trait under fixed levels of informed (green) settlement. Right panel zooms in on the scenarios of random settlement. informed ( $\circ$ ) departure combined with random (orange) or informed (green) settlement. 
bioRxiv preprint doi: https://doi.org/10.1101/267575; this version posted February 19, 2018. The copyright holder for this preprint (which was not certified by peer review) is the author/funder, who has granted bioRxiv a license to display the preprint in perpetuity. It is made available under aCC-BY-NC-ND 4.0 International license.

504 Figure 7: Local population variability for scenarios with fixed niche width. Scenarios of random $(x)$

505 and informed (०) departure combined with random (orange) or informed (green) settlement.

506

507 Table 1: fixed model parameters 


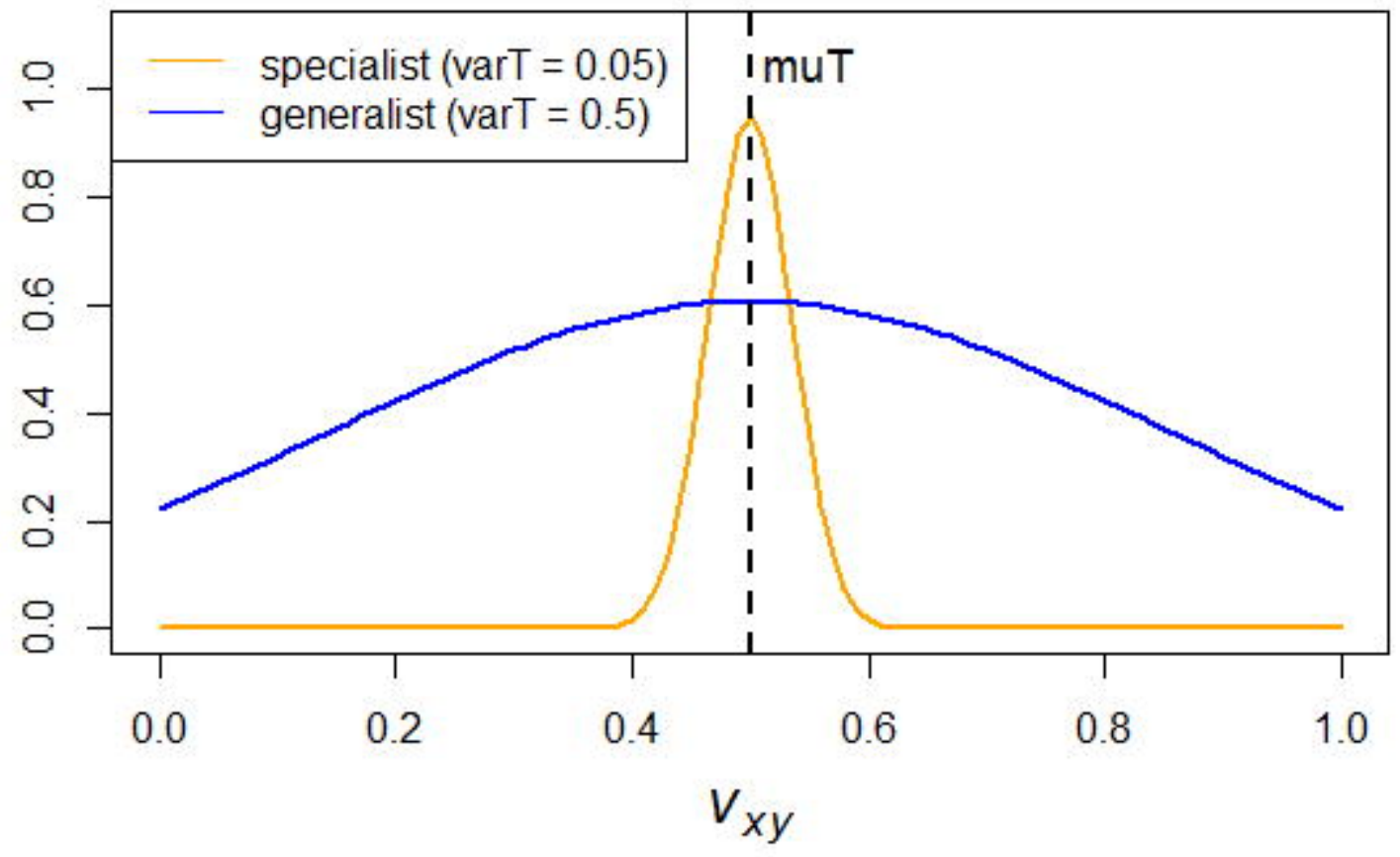




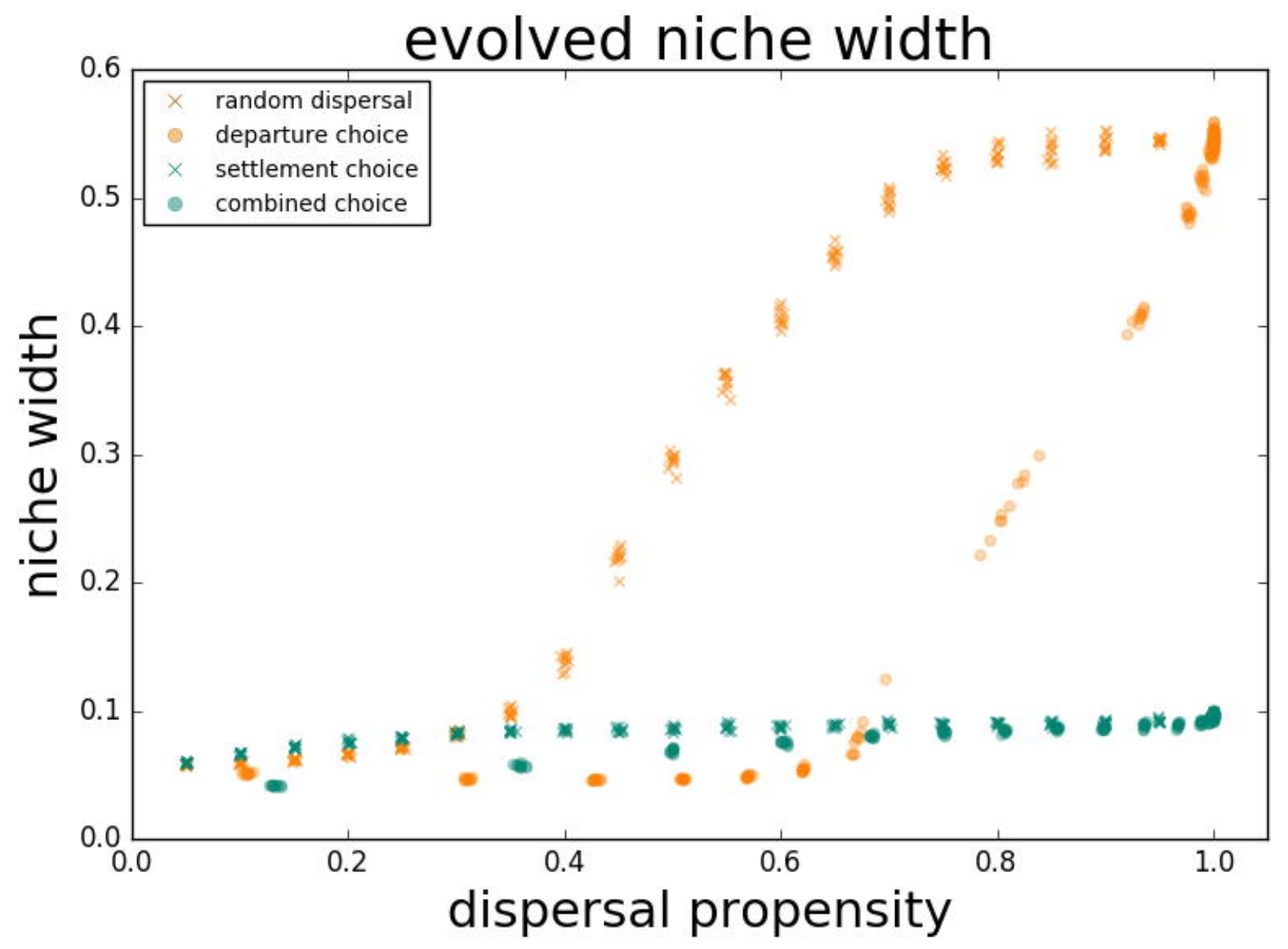




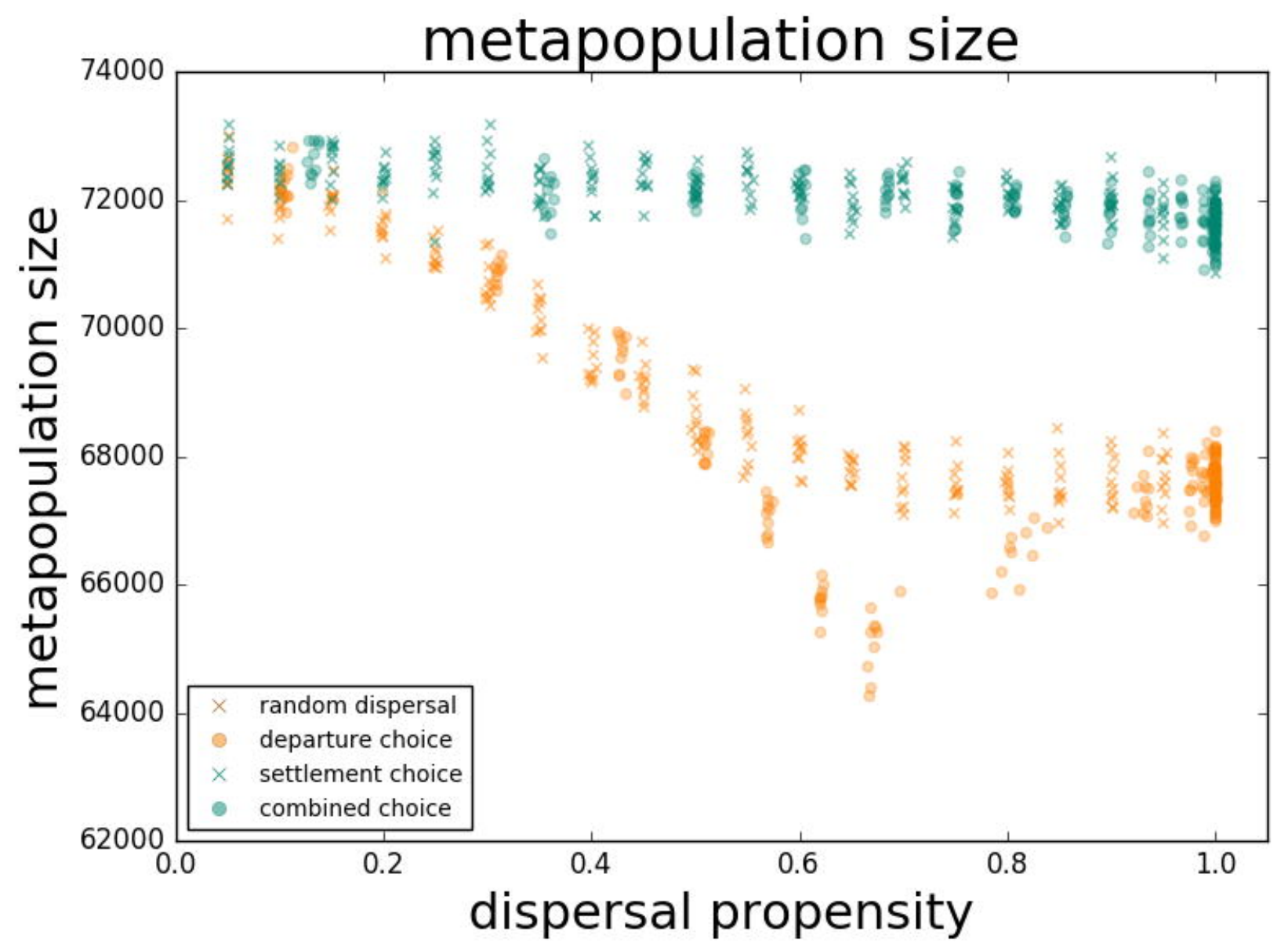




\section{local population variability}

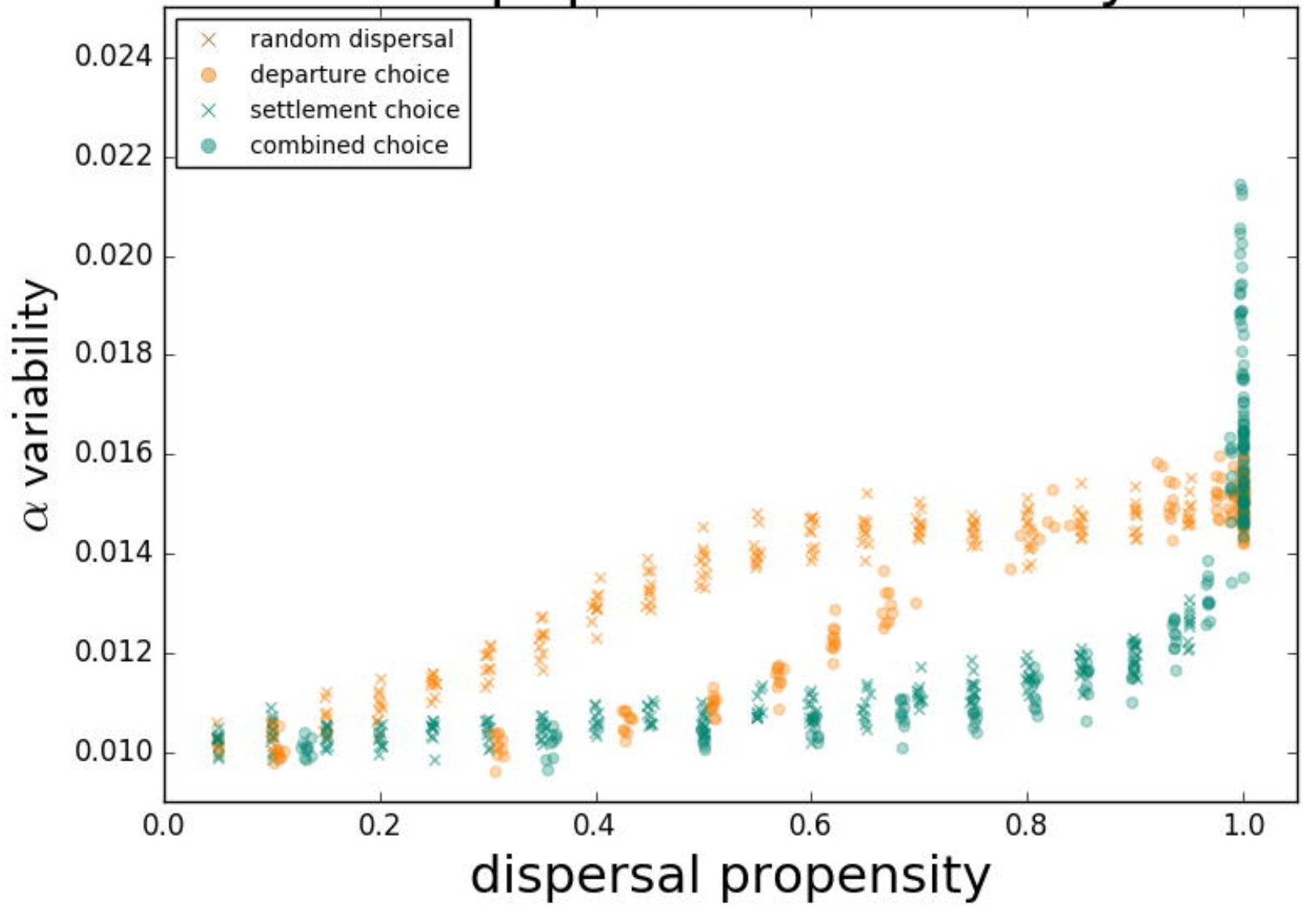




\section{dispersal propensity}

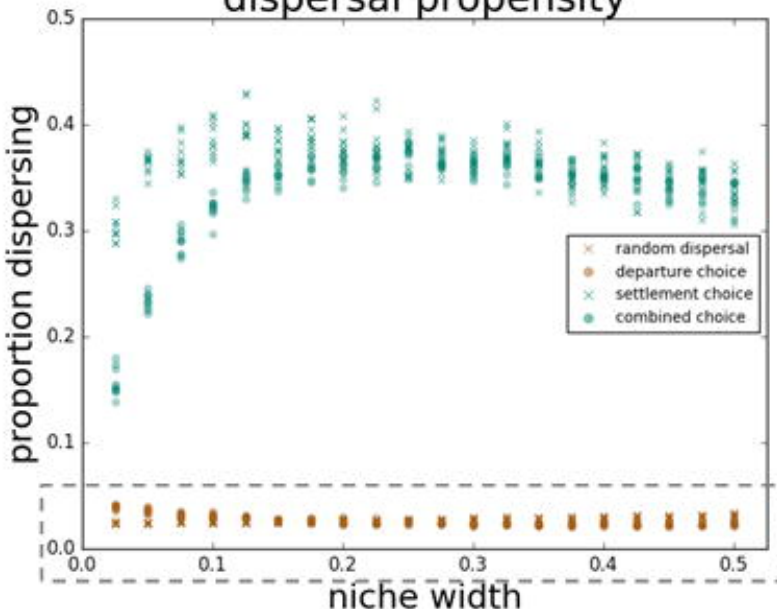

dispersal propensity

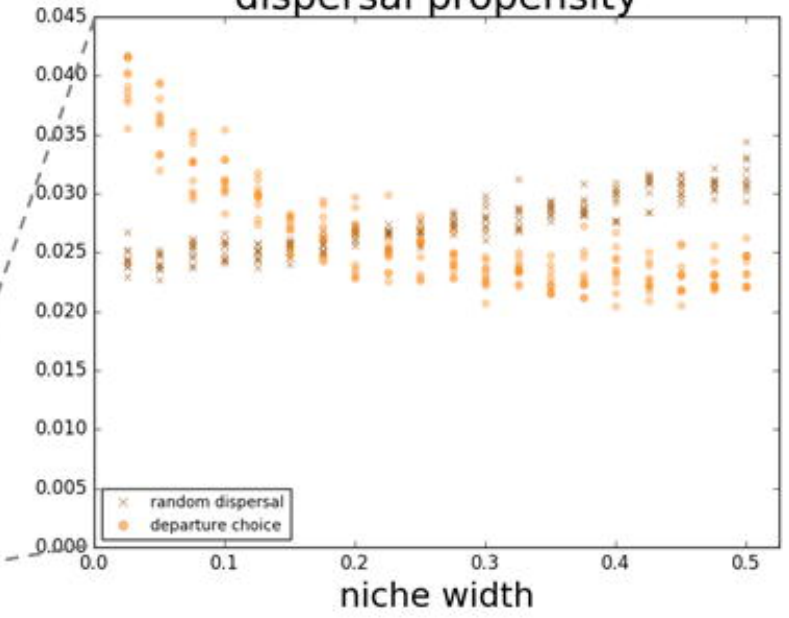




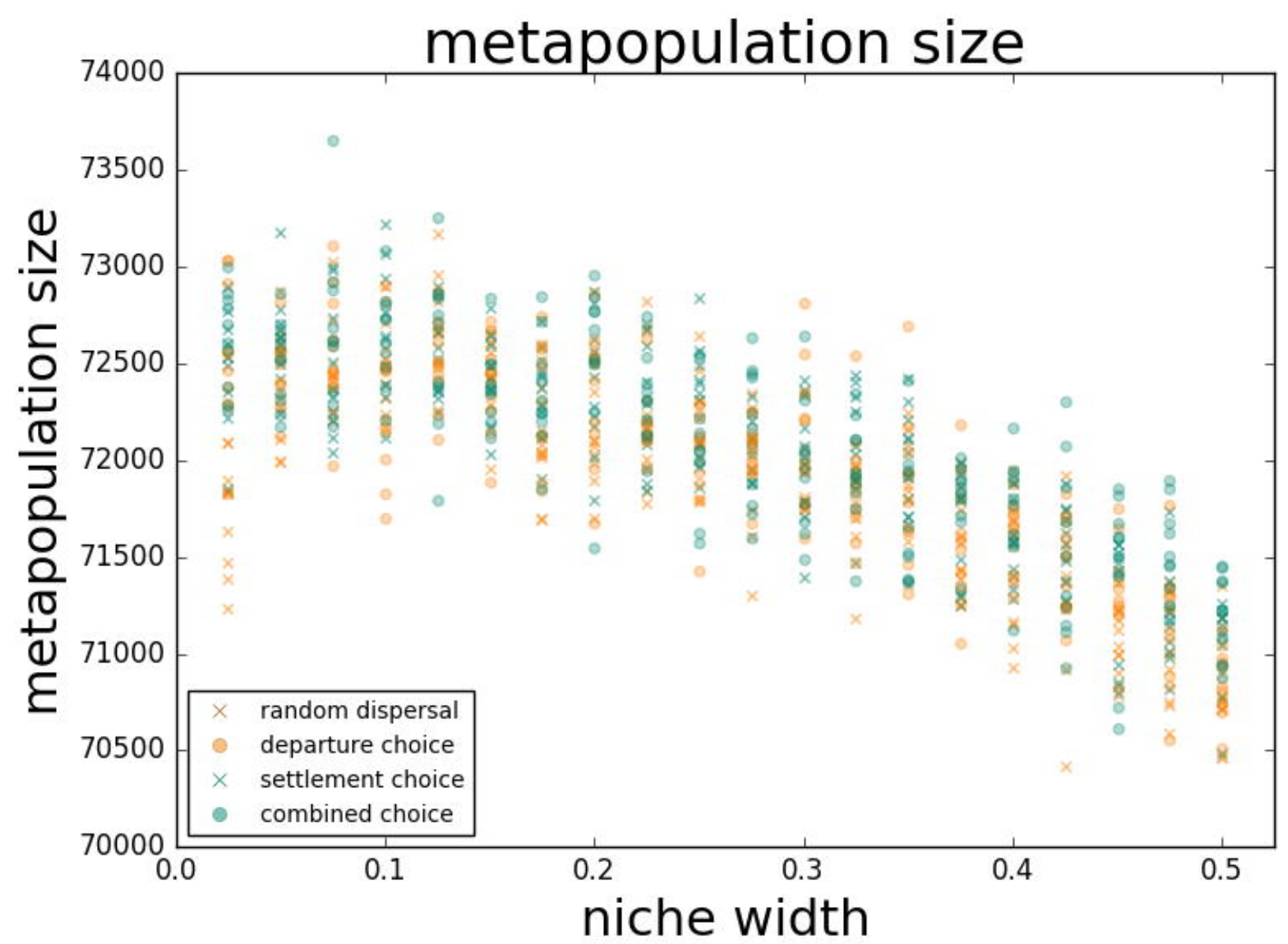




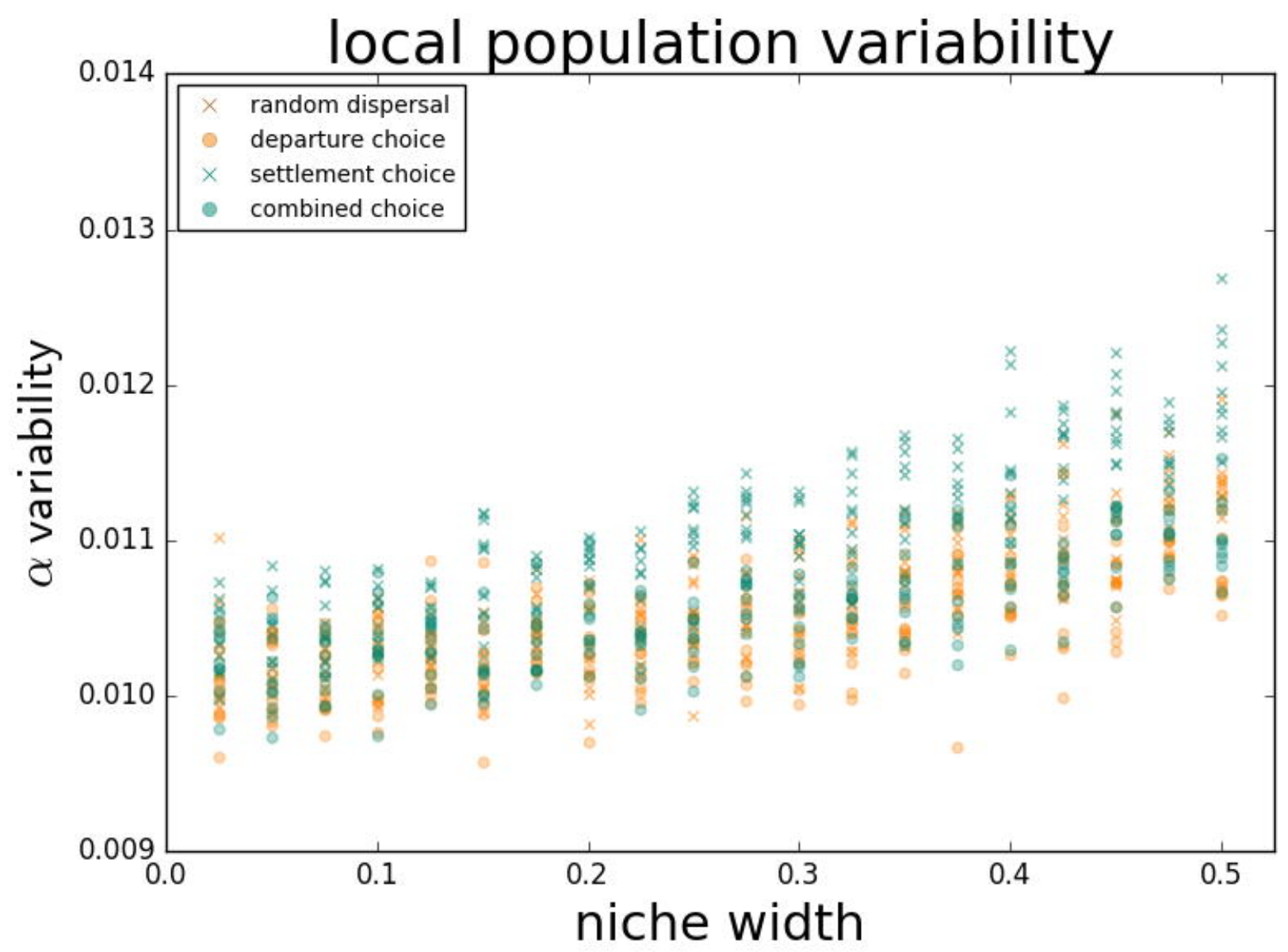

